Article

\title{
The Effect of Co-Monomer Content on the Swelling/Shrinking and Mechanical Behaviour of Individually Adsorbed PNIPAM Microgel Particles
}

\author{
Anna Burmistrova, Marcel Richter, Michael Eisele, Cagri Üzüm and Regine von Klitzing * \\ Stranski-Laboratorium für Physikalische und Theoretische Chemie, Institut für Chemie, Technische \\ Universität Berlin, Strasse des 17. Juni 124, D-10623 Berlin, Germany; \\ E-Mails: burmistrova@mailbox.tu-berlin.de (A.B.); marcel.richter@tu-berlin.de (M.R.); \\ miceis@ freenet.de (M.E.); uzum@mailbox.tu-berlin.de (C.Ü.) \\ * Author to whom correspondence should be addressed; E-Mail: klitzing@ mailbox.tu-berlin.de; \\ Tel.: +49-30-314-23476; Fax: +49-30-314-26602.
}

Received: 15 August 2011; in revised form: 23 August 2011 / Accepted: 20 September 2011 / Published: 26 September 2011

\begin{abstract}
The swelling/deswelling behaviour of microgel particles in the bulk and at the surface was studied and correlated to their mechanical properties. We focused on two kinds of particles: pure PNIPAM and PNIPAM-co-AAc particles. It was shown that the two step volume phase transition found for PNIPAM-co-AAc particles in the bulk disappears after the adsorption at the surface and only a one step transition was identified. The transition temperature increased strongly with increasing the co-monomer content. The dependence of the Young's modulus of the adsorbed microgel particles on the temperature and the co-monomer content was discussed. The investigations were performed via DLS and SFM.
\end{abstract}

Keywords: PNIPAM; microgels; AFM; elasticity; Young's modulus

\section{Introduction}

Polymers with "smart" behaviour are in the focus of a variety of research fields. One reason is their high impact for applications. Poly-N-isopropylacrylamide (PNIPAM) is particularly interesting due to its lower critical solution temperature (LCST) of around $32{ }^{\circ} \mathrm{C}$ in water [1,2]. Below this temperature 
the whole polymer network exists in a swollen state. On the other hand, by increasing the temperature above the LCST, the hydrophobic interactions dominate, resulting in a collapse of the polymer network. Due to this volume phase transition, PNIPAM attracted much attention in many application fields such as drug delivery, sensorics or in medicine [3-5]. Further on, the stimuli responsive behaviour of PNIPAM-based materials makes them interesting for coatings [6,7]. The ability of PNIPAM-based microgels to form well-organized structures at solid surfaces has been previously reported [8-12]. Schmidt et al. [8] demonstrated that pure PNIPAM microgel particles and particles containing acrylic acid as co-monomer form well-organized structures at solid surfaces. Microgel sensitivity to outer stimuli was also investigated for adsorbed microgel particles. It was shown that the film thickness made of microgels containing co-monomers can be controlled by the temperature and ionic strength, as well as the $\mathrm{pH}[12-14]$.

The properties of such PNIPAM based microgels can be tuned by incorporating co-monomers into the polymer network [15-17]. One type of commonly used co-monomers are organic acids such as vinylacetic, acrylic, methacrylic, allylacetic acid, etc. [1,17-20], which leads not only to thermo-responsive microgels but also to $\mathrm{pH}$ responsive ones. There are some theoretical and experimental publications studying the influence of the co-monomer type and concentration on swelling/deswelling behaviour of charged microgel particles [17-21]. Due to the deprotonation of carboxylic $(-\mathrm{COOH})$ groups, the interchain electrostatic repulsion and the increase in osmotic pressure lead to an increase in particle size $[1,12,18]$. Hoare and Pelton showed that highly ionized microgels have a sharp phase transition and that the LCST is shifted to higher temperatures compared to particles without co-monomer [17,19]. Kratz et al. [1] demonstrated the appearance of a two step volume phase transition for microgels with a very high co-monomer concentration. This phenomenon may be attributed to the competition between attractive hydrophobic and repulsive electrostatic forces.

The paper addresses the question whether a two-step transition is preserved at the surface after adsorption of the microgels and how different compartments of the microgel swell and shrink. Therefore, PNIPAM microgels containing acrylic acid as co-monomer with concentrations of $0 \%, 5 \%, 10 \%$ and $20 \%$ were synthesized and their thermo-induced swelling/deswelling behaviour of individually adsorbed microgels and microgels in the bulk was investigated. In order to follow the progress of swelling and shrinking in terms of structural changes, the Young's modulus of adsorbed microgels was determined. Adsorbed microgels were investigated by scanning force microscopy (SFM), while the particle behaviour in the bulk was studied by dynamic light scattering (DLS).

\section{Experimental Part}

\subsection{Microgel Synthesis}

The monomer N-isopropylacrylamide (NIPAM, Aldrich, 97\%), cross-linker N,N'methylenebisacrylamide (BIS, Aldrich, 99.5\%), the co-monomer acrylic acid (AAc, Aldrich, 99\%) and radical starter potassium persulfate (KPS, Fluka, 99\%) were used without further purification.

Microgels were synthesized via surfactant free emulsion polymerization [22]. NIPAM, AAc, BIS (the amount of BIS was fixed for all samples to $5 \mathrm{~mol} \%$ with respect to the amount of NIPAM) were dissolved in $200 \mathrm{~mL}$ Milli-Q water. The mixture was heated up to $70^{\circ} \mathrm{C}$ under a nitrogen atmosphere. To 
form the emulsion, the mixture was stirred with a rotation speed of $600 \mathrm{rpm}$. After one hour, $1.5 \mathrm{mg}$ KPS was dissolved in $1 \mathrm{~mL}$ Milli-Q water and rapidly added to the reaction flask. The transparent emulsion became turbid within $10 \mathrm{~min}$ after adding KPS. After $4 \mathrm{~h}$ the reaction was switched off via cooling down to room temperature. The cool solution was stirred under nitrogen atmosphere over night. To remove oligomers and unreacted monomers, microgels were cleaned by 14 days of dialysis in Milli-Q water. Water was exchanged every $24 \mathrm{~h}$. After cleaning, the microgels were freeze-dried. All synthesized microgels are listed in Table 1.

Table 1. Characteristics of the synthesized microgels. In the first column the names of the samples are presented. In the second, third and fourth columns the amount of NIPAM, BIS, AAc in grams is given. The molar percentage of BIS and AAc, with respect to the amount of NIPAM, is given in the fifth and the sixth columns, respectively. The effective amount of incorporated AAc units, determined by titration, is shown in the seventh column. The $\mathrm{pH}$ values for all used solution is shown in the last column.

\begin{tabular}{cccccccc}
\hline microgel type & NIPAM, g & BIS, $g$ & AAc, $g$ & BIS, \% & AAc, \% & eff. AAc, \% & pH value \\
\hline gel 0 & 2.26 & 0.154 & 0 & 5 & 0 & 0 & 5 \\
gel 5 & 2.26 & 0.154 & 0.072 & 5 & 5 & 3.3 & 5.3 \\
gel 10 & 2.27 & 0.154 & 0.144 & 5 & 10 & 5.8 & 5.6 \\
gel 20 & 2.26 & 0.154 & 0.289 & 5 & 20 & 15 & 4.79 \\
\hline
\end{tabular}

\subsection{Microgel Deposition}

Microgels were deposited on silicon wafers precoated by gold ( $\mathrm{Si} / \mathrm{Au}$ ) received from Georg Albert PVG-Beschichtungen via spin coating. For spin coating a SCS P6700 spin coater was used. In swelling experiments microgels were deposited from $10^{-3} \mathrm{M} \mathrm{NaCl}$ particle dispersion. The deposition duration was $300 \mathrm{~s}$ at 2,000 rpm for all samples. This preparation conditions assume a non-close packing of the microgels at the surface.

\subsection{Dynamic Light Scattering (DLS)}

Dynamic Light Scattering (DLS) measurements were carried out at a constant scattering angle of $75^{\circ}$ using an ALV goniometer setup. A fixed angle is justified due to the low polydispersity of the microgels. A Nd:YAG laser with a wave-length of $532 \mathrm{~nm}$, an output power $150 \mathrm{~mW}$ and an ALV-5000 correlator were used. In DLS measurements auto-correlation functions were recorded and analyzed by inverse Laplace transformation. The calculation was done by using the program CONTIN [23]. The heating/cooling rate was $2{ }^{\circ} \mathrm{C} / 30 \mathrm{~min}$.

\section{4. $\quad$ Scanning Force Microscopy (SFM)}

SFM measurements in liquids were performed in an ECCell (electrochemical cell) from JPK in the intermittent contact mode. For these measurements, uncoated silicon cantilevers CSC37 from Micromash were used. The temperature was charged from $20^{\circ} \mathrm{C}$ to $50^{\circ} \mathrm{C}$ in $5^{\circ} \mathrm{C}$ steps (long cycle) and from $20^{\circ} \mathrm{C}$ to $50^{\circ} \mathrm{C}$ in a $30^{\circ} \mathrm{C}$ step (short cycle). 
For particle characterization, their volume was calculated as

$$
V=\pi \int_{0}^{H} f^{2}(h) d h
$$

where $V$ is the particle volume, $H$ is the particle height, $f(h)$ is the particle cross-section. In order to minimize the effect of scanning rates and setpoint on the particles shape, particle cross sections were evaluated in vertical direction (parallel to the slow scan axis). In every SFM measurement, the particle volume was determined at least for three adsorbed microgel particles.

\subsection{Titration}

For titration of co-monomer microgel dispersions a Titrando 836 system from Metrohm was used. Titration was done by using a $25 \times 10^{-3} \mathrm{M} \mathrm{NaOH}$ solution. As shown in Table 1, not all AAc is incorporated into the polymer network. In the following the nominal AAc amount is referred to $0 \%, 5 \%$, $10 \%$ and $20 \%$.

\subsection{Elasticity Measurements of Microgels}

The Young's modulus $E$ was determined for the sample temperatures of 20 to $70^{\circ} \mathrm{C}$ in $5{ }^{\circ} \mathrm{C}$ steps as was previously described in detail [24]. All elasticity measurements were performed in liquid medium using an uncoated CSC 38 silicon cantilever (Micromash). Before starting the elasticity measurements, the cantilever deflection was calibrated in the same medium by a force measurement on a clean, hard surface. The calibration calculations were performed with the JPK SFM software package (JPK Instruments).

Force-indentation depth data were collected for at least three different particles using the SFM force mapping tool. Only the approach part of the force curves were used so that any adhesion in the retraction part would not affect the results. E was calculated for each indentation depth with a Hertz-based, film-thickness-corrected model suggested by Dimitriadis and co-workers [25]. This model considers $E$ as a function of force $F$, indentation depth $\delta$, film thickness $h$, indenter curvature $R$ and the Poisson's Ratio $v$. For incompressible hydrogels $(v=0.5)$ that are bonded to the substrate, $E$ can be calculated by:

$$
E=\frac{9 F}{16} \frac{1}{R^{1 / 2} \delta^{3 / 2}\left(1+1.133 \chi+1.283 \chi^{2}+0.769 \chi^{3}+0.0975 \chi^{4}\right)}
$$

where $\chi=\sqrt{R \delta} / h$. Data handling was achieved by self-made procedures on Igor Pro software package (Wavemetrics, Inc., USA). The height of the hydrogel particles $h$ was determined by SFM as discussed above. The indenting part of the SFM tips were assumed to be spherical and its exact radius of curvature $R$ was extracted from scanning electron microscope (SEM) images. The tip-sample contact point and the contribution of the electrostatic interactions on the force curves were determined by fitting the force data in the indentation regime and extrapolating it to the non-contact regime as was explained previously [24]. The error bars, which are shown in the relevant graphs, come from the calculation for at least three particles. 


\section{Results and Discussions}

\subsection{Microgel Characterizations in Bulk}

Firstly, the behaviour of microgel particles in bulk was investigated. Figure 1 demonstrates the dependence of hydrodynamic radius on temperature measured by DLS for four types of microgel particles: (a) pure PNIPAM, (b) with $5 \%$, (c) $10 \%$ and (d) $20 \%$ of AAc co-monomer (nominal AAc percentage, see Table 1).

Figure 1. Temperature dependence of the hydrodynamic radii of microgel particles with $0 \%$ (a); $5 \%$ (b); $10 \%$ (c); and 20\% (d) AAc contents measured by DLS. The temperature values refer to the volume phase transition during the heating (squares) and cooling (circles) process, respectively.
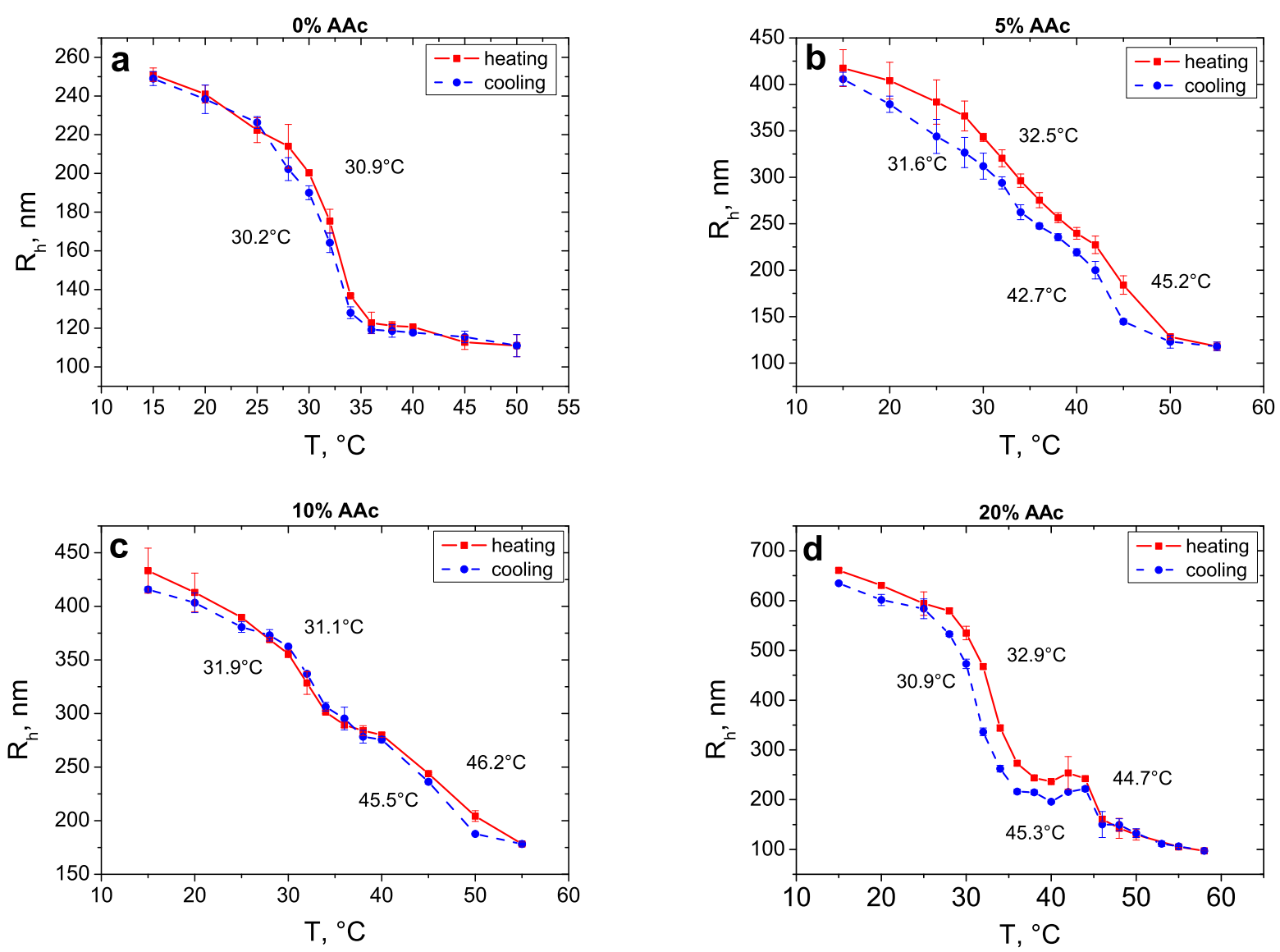

For all types of microgels the thermo-induced swelling and shrinking are completely reversible and a slight shift in the LCST takes place in cooling and heating processes (Table 2). As it was expected, the hydrodynamic radius increases with increasing AAc content because of the increasing electrostatic repulsion between the polymer chains and an increase in the osmotic pressure.

Pure PNIPAM microgels demonstrate a sharp transition at the temperature of about $30-31{ }^{\circ} \mathrm{C}$, which is in good agreement with the literature $[18,19,26]$. With increasing AAc content, the second transition step becomes more pronounced as already described in literature. Microgels with the highest AAc content (20\%) exhibit two very well separated and resolved transition steps. The first transition takes place at a temperature of about $32^{\circ} \mathrm{C}$, which results from the PNIPAM component caused by hydrophobic 
attraction related to the dehydration of the gel. Due to charge repulsion and enhanced osmotic pressure the phase transition is not completed at $32{ }^{\circ} \mathrm{C}$ but at $45^{\circ} \mathrm{C}$. The swelling ratio decreases with increasing co-monomer content as shown in Table 2.

Table 2. Characteristics of microgel particles with $0 \%, 5 \%, 10 \%$ and $20 \%$ AAc in bulk: hydrodynamic radius $\left(\mathrm{R}_{h}\right)$ at $20^{\circ} \mathrm{C}$ and at $50{ }^{\circ} \mathrm{C}$ and transition temperatures in heating and in cooling cycles. The last column shows the swelling ratio at $50^{\circ} \mathrm{C}$.

\begin{tabular}{|c|c|c|c|c|c|c|c|}
\hline \multirow{2}{*}{$\begin{array}{c}\text { AAc } \\
\text { content, \% }\end{array}$} & \multicolumn{2}{|c|}{$\mathbf{R}_{h}, \mathbf{n m}$} & \multicolumn{4}{|c|}{ transition temperature, ${ }^{\circ} \mathrm{C}$} & \multirow{2}{*}{$\begin{array}{c}\text { swelling ratio } o \\
\left(\mathrm{R}_{h 50} / \mathbf{R}_{h 20}\right)^{3}\end{array}$} \\
\hline & $20^{\circ} \mathrm{C}$ & $5^{\circ}{ }^{\circ} \mathrm{C}$ & \multicolumn{2}{|c|}{ heating } & \multicolumn{2}{|c|}{ cooling } & \\
\hline 0 & 241 & 111 & \multicolumn{2}{|c|}{30.9} & \multicolumn{2}{|c|}{30.2} & 0.1 \\
\hline 5 & 404 & 128 & 32.5 & 45.2 & 31.6 & 42.7 & 0.03 \\
\hline 10 & 413 & 204 & 31.1 & 46.2 & 31.9 & 45.5 & 0.1 \\
\hline 20 & 631 & 130 & 32.9 & 44.7 & 30.9 & 45.3 & 0.01 \\
\hline
\end{tabular}

\section{2. $\quad$ Swelling Behaviour of Adsorbed Microgel Particles}

In order to investigate the swelling/deswelling behaviour of adsorbed PNIPAM microgels, SFM measurements were performed in a temperature controlled liquid cell. Figure 2 shows the SFM images of all four types of microgel particles deposited on Au-coated Si-wafers at $20^{\circ} \mathrm{C}$ (swollen state) and at $50^{\circ} \mathrm{C}$ (collapsed state). The particle size increases with increasing the amount of co-monomer as already discussed for the bulk. It should be noted that the particle pattern does not change during the heating process and no particle desorption takes place.

Figure 2. SFM height images of microgel particles with $0 \%(\mathbf{a}, \mathbf{b}), 5 \%(\mathbf{c}, \mathbf{d}), 10 \%(\mathbf{e}, \mathbf{f})$ and $20 \%(\mathbf{g}, \mathbf{h})$ AAc content deposited on gold wafer. Images were taken in SFM liquid cell at $20^{\circ} \mathrm{C}(\mathrm{a}, \mathrm{c}, \mathrm{e}, \mathrm{g})$ and at $50^{\circ} \mathrm{C}(\mathrm{b}, \mathrm{d}, \mathrm{f}, \mathrm{h})$. Scan area: $5 \times 5 \mu \mathrm{m}^{2}$.

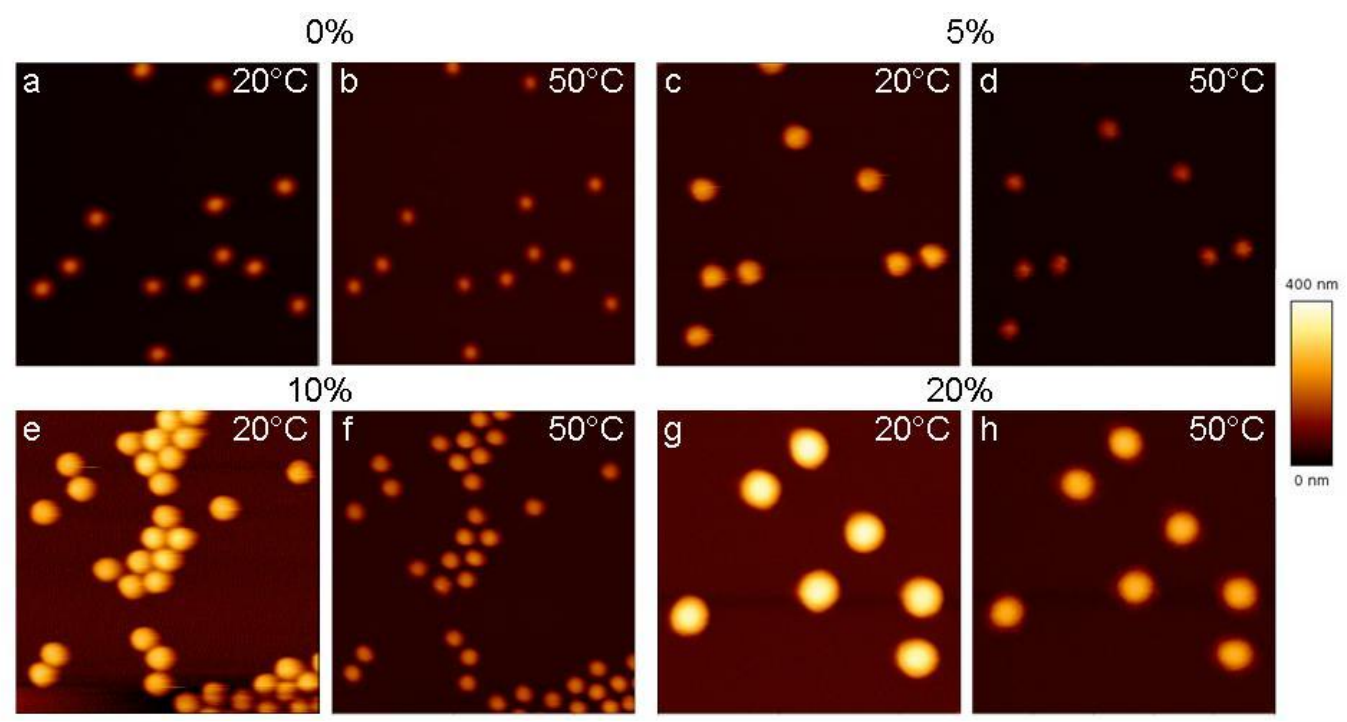

In order to compare the behaviour of deposited microgels, the cross sections of particles, which were obtained from the particles in Figure 2, with $0 \%, 5 \%, 10 \%$ and $20 \%$ AAc in the swollen $\left(20^{\circ} \mathrm{C}\right)$ and collapsed states $\left(50^{\circ} \mathrm{C}\right)$ in two short cycles are presented in Figure 3. All adsorbed microgels 
demonstrate a reversible thermo-induced swelling/shrinking behaviour. The size change of adsorbed microgels mainly takes place in a vertical direction.

Figure 3. Cross sections of microgel particles with (a) 0\%; (b) 5\%; (c) 10\%; and (d) 20\% AAc contents adsorbed on gold wafer measured by SFM at two short cycles: $20^{\circ} \mathrm{C}, 50^{\circ} \mathrm{C}$, $20^{\circ} \mathrm{C}, 50{ }^{\circ} \mathrm{C}$ and $20^{\circ} \mathrm{C}$.
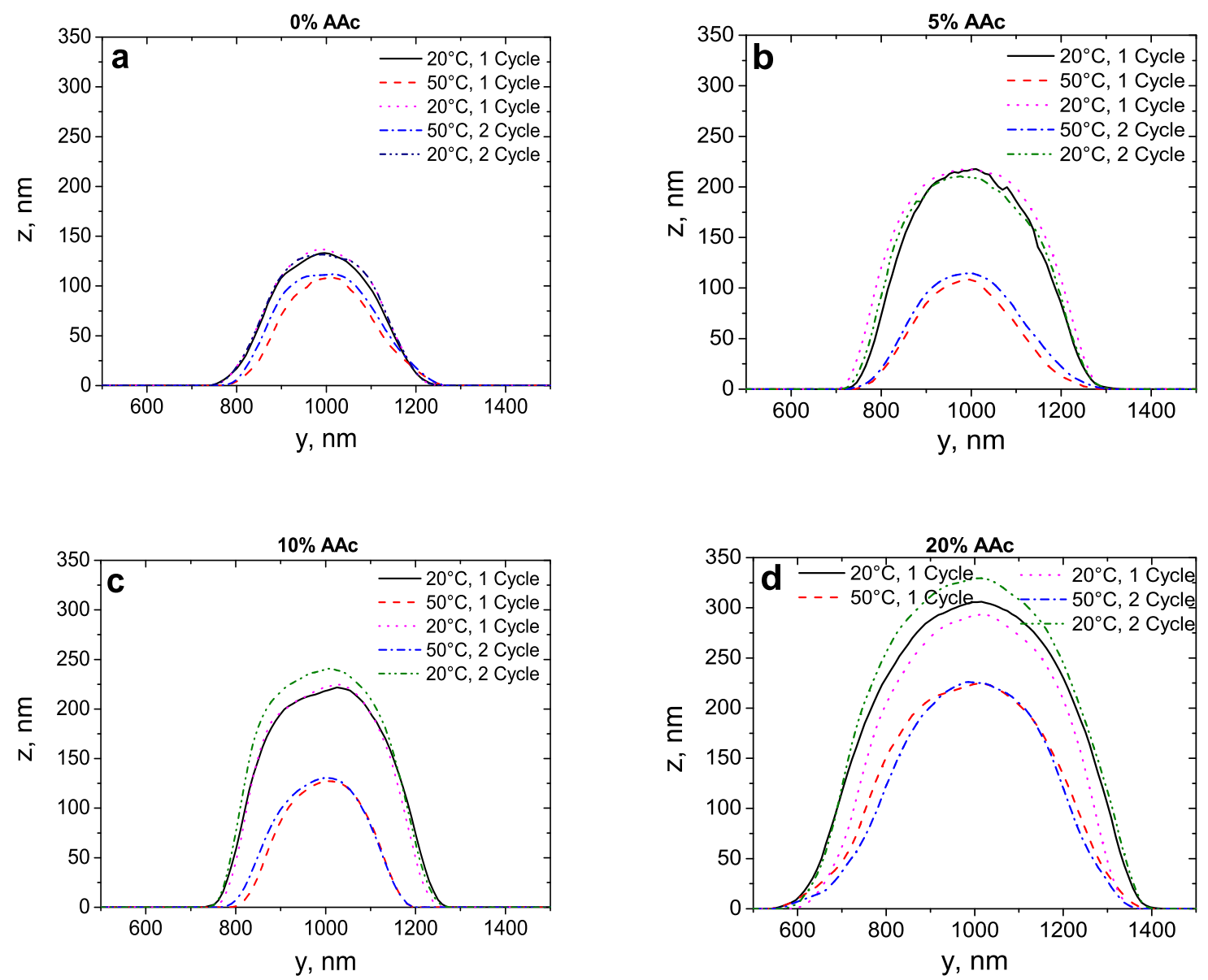

For a better understanding of the swelling/deswelling behaviour of adsorbed microgels with different AAc contents, one long cycle was carried out. Based on the cross section data, Figure 4 demonstrates the volume change of adsorbed microgels as a function of temperature.

Adsorbed microgels demonstrate a reversible thermo-induced swelling/shrinking behaviour. A slight shift in the LCST takes places in cooling and heating processes (Table 3).

In contrast to the behaviour in the bulk, all four microgel types have just one transition temperature after the adsorption. With increasing co-monomer content, the LCST shifts to higher temperatures. The LCST of pure PNIPAM microgels (0\% AAc) and the ones with extremely high AAc concentration (20\%) are sharper compared to those of the particles with 5\% and 10\% AAc. Adsorbed pure PNIPAM microgels and microgels in bulk have the LCST at the same temperature of $30^{\circ} \mathrm{C}$ (Tables 2 and 3). The transition temperature of deposited microgels with $20 \%$ AAc arises at around $45^{\circ} \mathrm{C}$. It corresponds to the second transition of microgels in bulk affected by a high co-monomer concentration. Particles with an AAc concentration of $5 \%$ and $10 \%$ have a broad phase transition at temperatures between $34{ }^{\circ} \mathrm{C}$ and $37^{\circ} \mathrm{C}$. Due to the increase in the charge density, the phase transition at $32{ }^{\circ} \mathrm{C}$ disappears for the microgel with $20 \%$ AAc. In case of the particles synthesised with 5\% and 10\% AAc, one can observe 
a combination of the two phase transitions caused by PNIPAM. In case of adsorbed particles, the higher charge density compared to those in the bulk affects a strong intrachain repulsion. Microgels in the bulk contain PNIPAM subcompartments, which lead to a collective swelling/shrinking behaviour.

Figure 4. Temperature dependence of volume of microgel particles with $0 \%$ (a); 5\% (b); $10 \%$ (c); and $20 \%$ (d) AAc contents deposited on gold wafer measured by SFM.
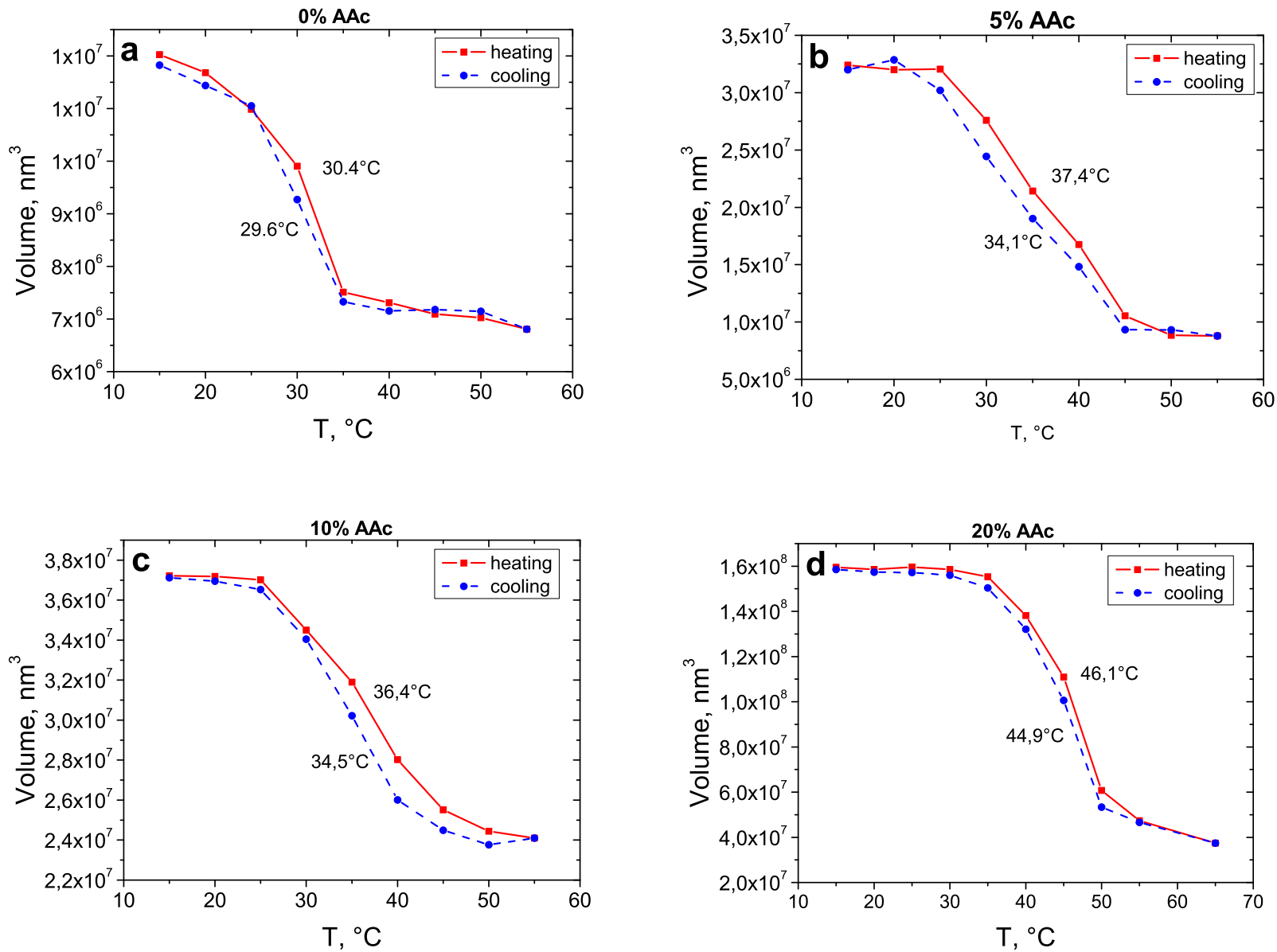

Table 3. Characteristics of adsorbed microgel particles for microgels with $0 \%, 5 \%, 10 \%$ and 20\% AAc contents: LCST in heating and cooling cycles, volume of deposited microgels in dry state and in liquid at $20^{\circ} \mathrm{C}$ and $50{ }^{\circ} \mathrm{C}$, and particle volume in liquid cell $\left(\mathrm{V}^{T}\right.$ ads $)$ at temperature $T$ normalized by volume of dried particles $\left(\mathrm{V}_{d r y}\right)$.

\begin{tabular}{|c|c|c|c|c|c|c|c|}
\hline \multirow{2}{*}{$\begin{array}{c}\text { AAc } \\
\text { content, \% }\end{array}$} & \multicolumn{2}{|c|}{ LCST, ${ }^{\circ} \mathrm{C}$} & \multicolumn{3}{|c|}{ Volume, $\times 10^{6} \mathrm{~nm}^{3}$} & \multicolumn{2}{|c|}{$\mathrm{V}^{T}{ }_{a d s} / \mathrm{V}_{d r y}$} \\
\hline & heating & cooling & dried & $20{ }^{\circ} \mathrm{C}$ & $50{ }^{\circ} \mathrm{C}$ & $\mathrm{T}=20^{\circ} \mathrm{C}$ & $\mathbf{T}=\mathbf{5 0}{ }^{\circ} \mathrm{C}$ \\
\hline 0 & 30.4 & 29.6 & 5.34 & 11.7 & 7.02 & 2.2 & 1.3 \\
\hline 5 & 37.4 & 34.1 & 5.76 & 32.0 & 8.84 & 5.6 & 1.5 \\
\hline 10 & 36.4 & 34.5 & 6.95 & 37.2 & 24,4 & 5.4 & 3.5 \\
\hline 20 & 46.1 & 44.9 & 32.9 & 159 & 60.8 & 4.8 & 1.8 \\
\hline
\end{tabular}

The phase transition is not complete at $32^{\circ} \mathrm{C}$ as the charge repulsion and the osmotic pressure counteract a total collapse up to $45^{\circ} \mathrm{C}$. At the surface the particles are more compressed compared to the ones in the bulk and the PNIPAM network is completely riddled with co-monomers, which suppresses the ability of pure PNIPAM gels. By increasing the temperature up to $32{ }^{\circ} \mathrm{C}$, the shrinking process of 
PNIPAM starts, but is hindered due to the functional groups resulting in an incomplete network collapse. Finally, the hydrophobic interactions overcome the electrostatic repulsion caused by the co-monomer and the polymer network reaches its final size. Microgels with the highest co-monomer content (20\%) exhibit an even more concentrated amount of carboxyl groups, which results in the suppression of the PNIPAM collapse. Only the sharp phase transition of the polymer network can be observed at $45^{\circ} \mathrm{C}$. This process is depicted in Figure 5. In contrast to the effect of cross linker [24], the amount of co-monomer has almost no influence on the hysteresis.

Figure 5. A: Particles in the bulk: the morphology suggests a lower charge density and gives a possibility for a PNIPAM collapse. The coloured areas correspond to undisturbed PNIPAM microdomains, which overlap and are riddled with co-monomers after adsorption, B: Adsorbed particles: the functional groups exhibit a higher charge density resulting in hindered or disappeared PNIPAM collapse.
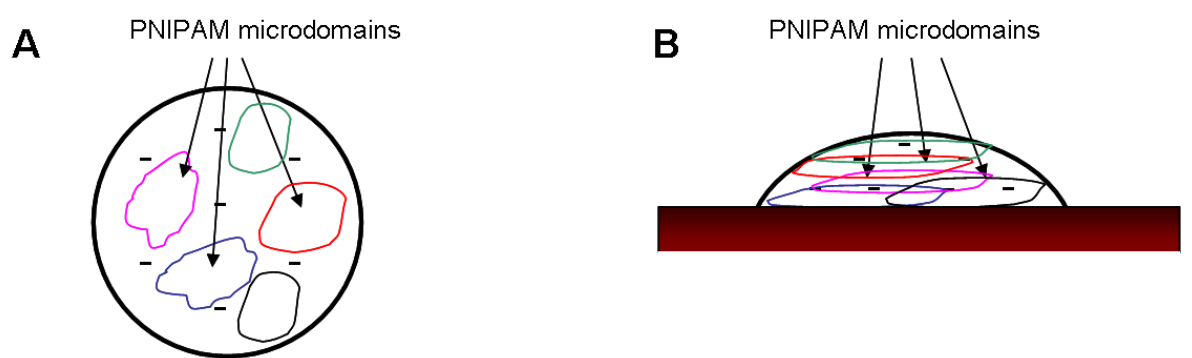

In context of the reversibility of the swelling behaviour, the reswelling of the particles was studied after a complete drying. In Figure 6 the volume of deposited microgels in the swollen and in the collapsed state in long cycle (l.c.) and in two reswelling cycles (r.c.) is presented. Reswelling cycles demonstrate the thermo-induced behaviour of adsorbed particles after the complete drying. The microgels still exhibit sensitivity to temperature changes and show reversible swelling/deswelling behaviour. The reswollen microgels maintain the same volume values as before drying.

Figure 6. Temperature dependence of volume of microgel particles with $0 \%$ (squares), $5 \%$ (circles), $10 \%$ (triangles) and 20\% (rhombs) AAc content deposited on Si/Au measured by SFM in one long cycle (l.c.) and in two reswelling cycles (r.c.).

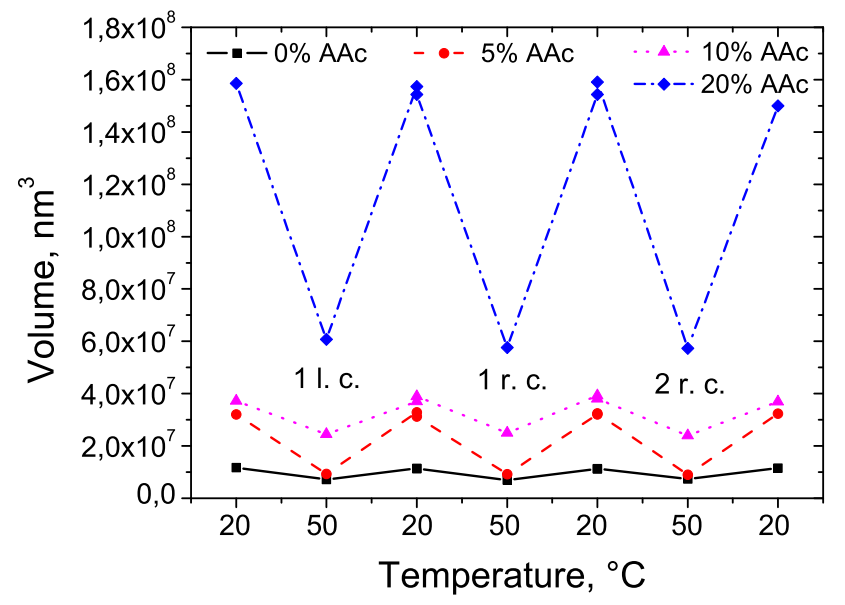


Microgels in bulk and deposited at solid surface demonstrate qualitatively similar behaviour with respect to the reversibility of the phase transition (Figure 7). In both geometries the particle size increases with increasing AAc concentration. Due to the attractive interactions between the substrate and both the deprotonated co-monomer and the radical starter, the particle volume at the substrate is one order of magnitude lower than that in the bulk.

Figure 7. Temperature dependence of microgel particle volume (a) in bulk and (b) deposited on gold synthesised with $0 \%$ (squares), 5\% (circles), 10\% (triangles) and 20\% (rhombs) AAc contents measured by DLS and SFM, respectively.
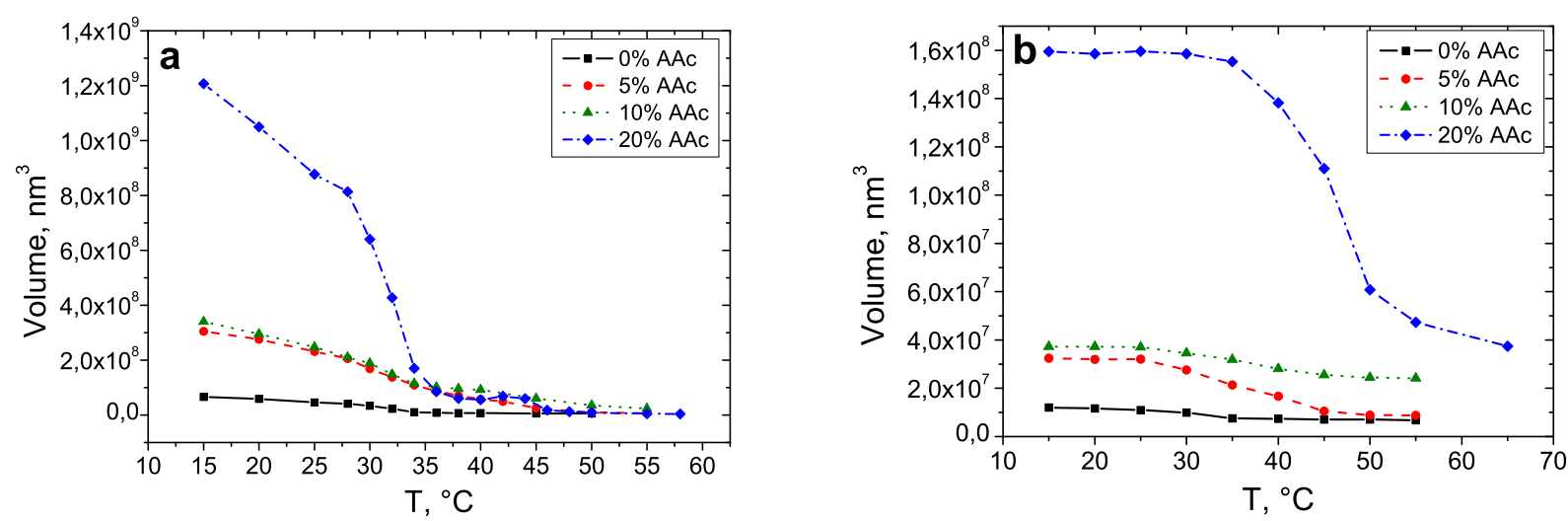

\subsection{Elasticity Measurements}

After scanning with SFM, a grid was placed over the imaged particles, as shown in Figure 8.

Figure 8. JPK force mapping tool. Every square represents a force curve with its extended and retracted part. For the calculation the number-marked squares were used.

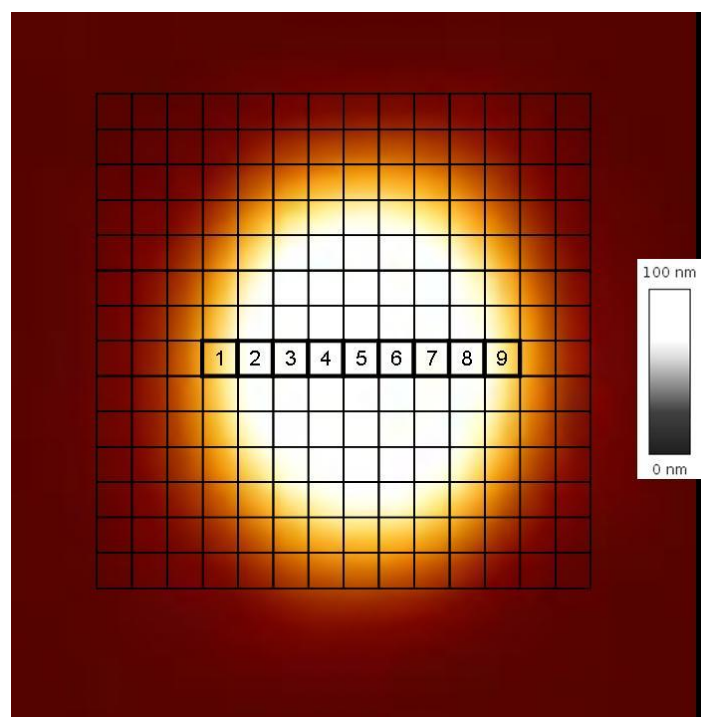

At each square a force curve with its approach and retraction part was recorded. For the analysis only the approach curves were used, since the adhesion is extremely strong during retraction. Hashmi et al. [27] showed that the Young's modulus does not depend significantly on the measurement 
velocity so possible viscous behaviour of the particles was neglected throughout this study. The numbered squares in Figure 8 were used for the determination of the Young's modulus in a temperature range from 20 to $70^{\circ} \mathrm{C}$.

Figure 9 shows the Young's modulus at different cross section positions for particles synthesised with $20 \%$ AAc at $20{ }^{\circ} \mathrm{C}$ and $70{ }^{\circ} \mathrm{C}$. The Young's modulus increases and decreases in accordance to the particle height as was reported before [24]. For both temperatures, the stiffness decreases from the centre to the edges of the microgel. This result is in good agreement with the literature [19] since a core-shell structure is introduced for the particle morphology with its higher cross-linked core and the lower cross-linked shell. The stiffness doubles itself from 20 to $70{ }^{\circ} \mathrm{C}$. Since the Young's modulus was determined for at least three particles, the pronounced error bars in the centre of the microgel indicate an inhomogeneous microgel character. This suggests that the inner morphology differs from particle to particle.

Figure 9. Height and Young's modulus versus the cross section. Top: microgel synthesised with $20 \%$ AAc at $20^{\circ} \mathrm{C}$, bottom: same particle at $70^{\circ} \mathrm{C}$. It is shown that the Young's modulus follows the cross section height and increases with temperature.
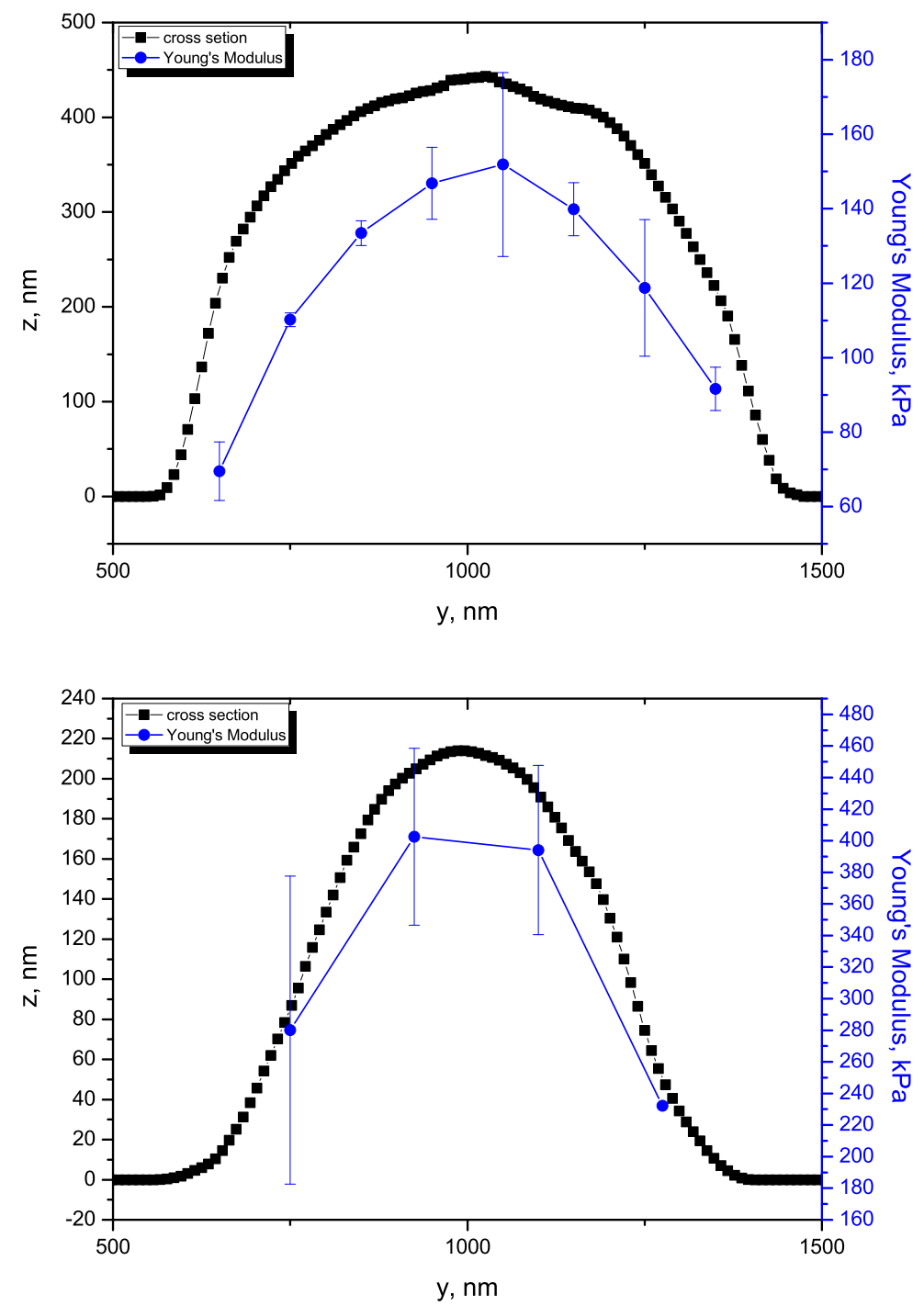
Figure 10 shows the temperature dependence of the Young's modulus of the microgels synthesised with $0 \%$ (squares) and 20\% (circles) AAc at the centre of the particle (square no. 5 corresponding to Figure 8). At low temperatures the stiffness of both microgels is equal and confirm the previous elasticity measurements on PNIPAM films [28]. The periphery morphology for both microgels should be equal resulting in the same Young's modulus. With increasing temperature, the stiffness of both pure PNIPAM and PNIPAM-co-AAc particles increases, due to the shrinking process dominated by hydrophobic interactions. While the stiffness of the microgel synthesised with $20 \%$ AAc slightly enhanced, PNIPAM particles without co-monomer end up with a higher Young's modulus. It should be noted that in elasticity measurements, the LCST of the microgel containing $20 \%$ AAc shifts to higher temperature compared to the one obtained by SFM and DLS measurements. The transition temperature shifts by about $10^{\circ} \mathrm{C}$ from $45^{\circ} \mathrm{C}$ ( SFM cross section) to $55^{\circ} \mathrm{C}$ (Young's modulus). In the case of pure PNIPAM particles, a slight LCST shift takes place from $31^{\circ} \mathrm{C}$ to $36^{\circ} \mathrm{C}$. These results are consistent with a core-shell model introduced by Daly and Saunders [29]. They developed a model with a stepwise collapse of the polymer network. At first, the higher cross-linked core collapses. By further heating the shell expels water, resulting in a more pronounced shrinking. Force measurements confirm this core-shell model by Daly and Saunders. At low temperature the AFM tip penetrates the lower cross-linked shell. Due to the hydration of the polymer network, the microgel is extended leading to a low Young's modulus. With the increase in temperature, the core starts the shrinking process, while the shell keeps its original size. This leads to a constant Young's modulus as the AFM tip still measures the elasticity of the lower cross-linked shell. Further heating leads to the shell collapse resulting in a tremendous increase in the measured stiffness of the PNIPAM particles and a less pronounced increase for PNIPAM-co-AAc. The Young's modulus and size stay constant after the shell collapse.

Figure 10. Temperature dependence of the Young's modulus. Squares represent particles without co-monomer, circles represent the microgel with $20 \%$ AAc. Young's modulus increases with increasing the temperature. At low temperature, both microgels with $0 \%$ and $20 \%$ AAc have the same Young's modulus. At higher temperatures, the microgel synthesised with $20 \%$ AAc is much softer than that without co-monomer due to the repulsive interactions.

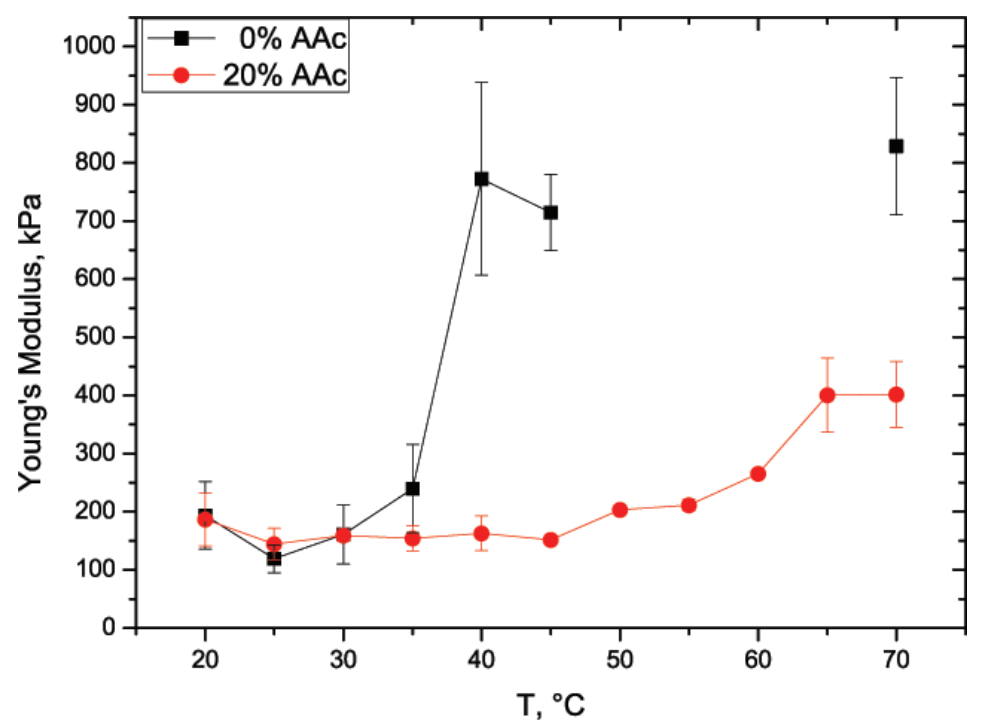


This model also explains the LCST shift for both PNIPAM and PNIPAM-co-AAc microgels, shown in Figure 11. The higher Young's modulus and the slightly larger LCST value of the pure PNIPAM gels compared to the gels containing AAc suggests a less pronounced core-shell structure in the former. Due to the disappearance of the co-monomer in the case of pure PNIPAM, a "smaller" shell is formed during the synthesis, which results in a less pronounced core-shell structure. On the other hand, the incorporation of the co-monomer causes an increase in both the diffuse shell and the mesh size, which results in a softer microgel at high temperatures and a more expressed core-shell structure of those particles. The soft behaviour of PNIPAM-co-AAc particles also results from a high water content in the polymer network even at temperatures above the LCST. Schmidt et al. showed just a slight shift in the refractive index from 1.34 (below LCST) to 1.37 (above LCST) [13]. Neutron experiments confirm a water content up to $90 \%$ in such gels [30]. The core-shell structure becomes even more pronounced by the fact that the charges are expelled towards the shell of the particles [17,19]. Zeta potential measurements show that most of the functional groups are located in the particle periphery [12]. With the collapse of the polymer network, the charge density increases in the shell, resulting in a softening of the particle.

Figure 11. Shrinking process of the microgel containing 20\% AAc. The black curve represents the particle volume and the blue line corresponds to the Young's modulus: (1) swollen state: the particle exhibits its largest size and lowest Young's modulus, (2) core shrinking process: the size decreases slightly, while the elasticity stays more or less constant, (3) shell collapse: a further reduction in size and an enhancement in stiffness take place.

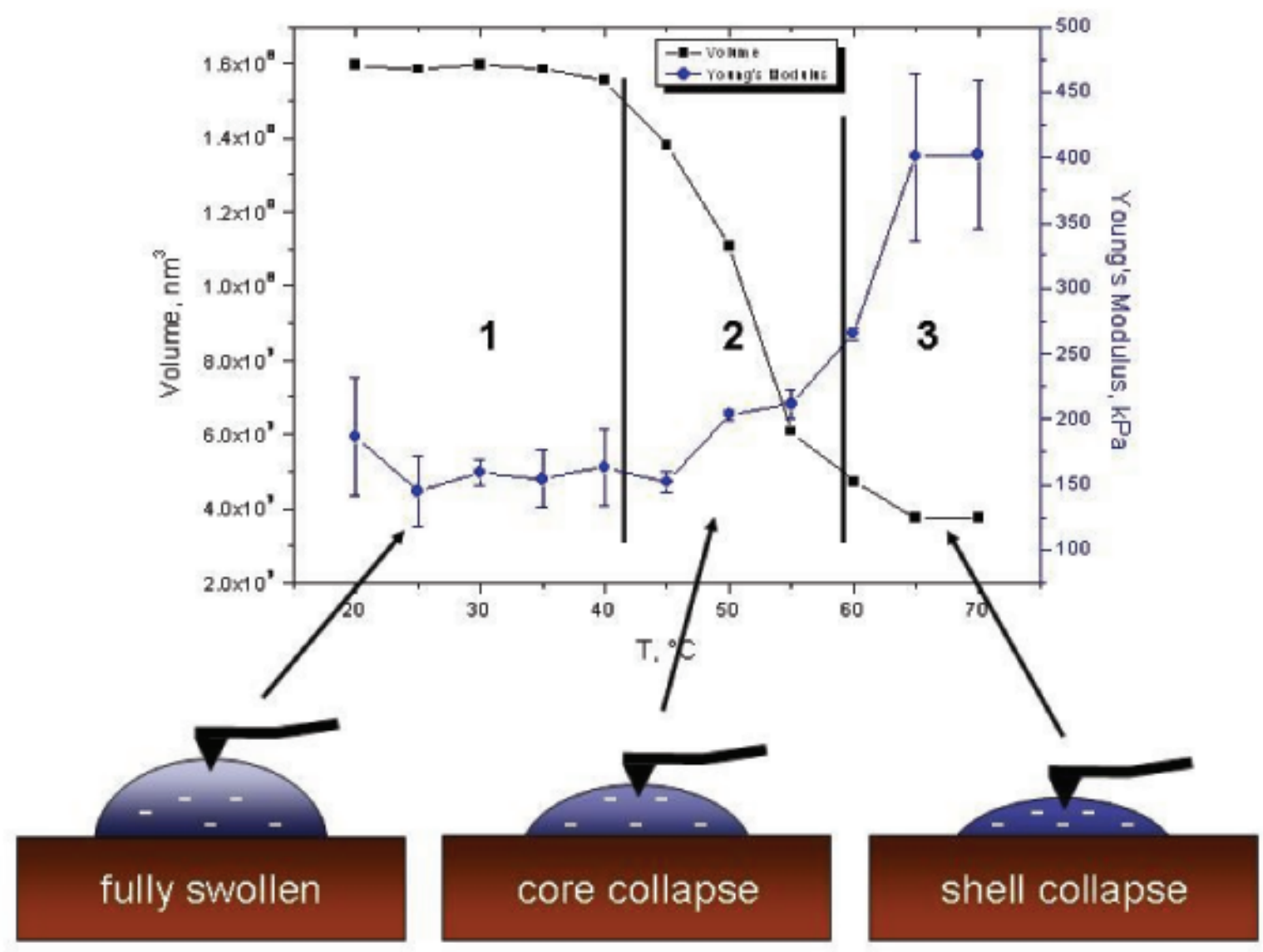




\section{Conclusions}

This paper deals with PNIPAM and PNIPAM-co-AAc microgels, which differ in the co-monomer content. The swelling/shrinking behaviour of these particles was investigated in the bulk and at the surface. For the adsorbed particles the Young's modulus was also studied.

It was shown that pure PNIPAM particles collapses completely at $32{ }^{\circ} \mathrm{C}$, while the particles containing AAc exhibit a two phase transitions at $32^{\circ} \mathrm{C}$ and $45^{\circ} \mathrm{C}$. The second phase transition at $45^{\circ} \mathrm{C}$ is more pronounced at higher co-monomer content. In the bulk, the PNIPAM-co-AAc particles comprise PNIPAM subcompartments, which collapses at around $32^{\circ} \mathrm{C}$. The total collapse takes place at $45^{\circ} \mathrm{C}$, when the charges can no longer prevent the collapse of the gel particles. At the surface, the particles containing AAc exhibit only one LCST, which is shifted to higher temperatures compared to the LCST in the bulk. Due to the adsorption the particles are compressed, which leads to a higher charge density. This results in smaller undisturbed subcompartments of the adsorbed PNIPAM compared to the bulk, which leads to a mixed effect of PNIPAM collapse and electrostatic repulsion of the co-monomer and therefore to a continuous shift of the LCST. The Young's modulus showed a strong temperature dependence. The stiffness increases with increasing the temperature. The Young's modulus can be tuned by incorporating co-monomers. It was shown that PNIPAM-co-AAc microgels are softer than those ones without co-monomer. This indicates a distinctive core-shell structure of particles containing AAc. The LCST (Young's modulus) shifts to higher temperatures compared to the LCST (SFM), which also corresponds to the core-shell morphology of PNIPAM-co-AAc particles.

\section{Acknowledgement}

Financial support by the Deutsche Forschungsgemeinschaft (SPP1259 and IGTRG 1524) and TU Berlin is kindly acknowledged.

\section{References}

1. Kratz, K.; Hellweg, T.; Eimer, W. Influence of charge density on the swelling of colloidal poly(N-isopropylacrylamide-co-acrylic acid) microgels. Colloids Surf. A 2000, 170, 137-149.

2. Nayak, S. Soft nanotechnology with soft nanoparticles. Angew. Chem. Int. Ed. 2005, 44, 7686-7708.

3. Hoare, T.; Perlton, R. Impact of microgel morphology on functionalized microgel-drug interactions. Langmuir 2008, 24, 1005-1012.

4. Kim, J.; Nayak, S.; Lyon, L.A. Bioresponsive hydrogel microlenses. J. Am. Chem. Soc. 2005, 127, 9588-9592.

5. Kim, J.; Serpe, M.J.; Lyon, L.A. Photoswitchable microlens arrays. Angew. Chem. 2005, 117, 1357-1360.

6. Quint, S. Extraordinary long range order in self-healing non-close packed 2D arrays. Soft Matter 2011, 7, 3735-3738.

7. Zavgorodnya, O. Assembly of poly(N-isopropylacrylamide)-co-acrylic acid microgel thin films on polyelectrolyte multilayers: Effects of polyelectrolyte layer thickness, surface charge, and microgel solution pH. Colloid Polym. Sci. 2011, 289, 591-602. 
8. Schmidt, S.; Hellweg, T.; von Klitzing, R. Packing density control in P(NIPAM-co-AAc) microgel monolayers: Effect of surface charge, ph, and preparation technique. Langmuir 2008, $24,12595-12602$.

9. Lyon, L.A.; Debord, J.D.; Debord, S.B.; Jones, C.D.; McGrath, J.G.; Serpe, M.J. Microgel colloidal crystals. J. Phys. Chem. B 2004, 108, 19099-19108.

10. South, A.B.; Whitmire, R.E.; Garcia, A.J.; Lyon, L.A. Centrifugal deposition of microgels for the rapid assembly of nonfouling thin films. Appl. Mater. Interfaces 2009, 1, 2747-2754.

11. Hellweg, T.; Dewhurst, C.D.; Brueckner, E.; Kratz, K.; Eimer, W. Colloidal crystals made of poly(N-isopropylacrylamide) microgel particles. Colloid Polym. Sci. 2000, 278, 972-978.

12. Burmistrova, A.; von Klitzing, R. Control of number density and swelling/shrinking behavior of P(NIPAM-AAc) particles at solid surface. J. Mater. Chem. 2010, 20, 3502-3507.

13. Schmidt, S.; Motschmann, H.; Hellweg, T.; von Klitzing, R. Thermoresponsive surfaces by spin-coating of PNIPAM-co-PAA microgels: A combined AFM and ellipsometry study. Polymer 2008, 49, 749-756.

14. Nerapusri, V.; Keddie, J.L.; Vincent, B.; Bushnak, I.A. Swelling and deswelling of adsorbed microgel monolayers triggered by changes in temperature, $\mathrm{pH}$, and electrolyte concentration. Langmuir 2006, 22, 5036-5041.

15. Al-Manasir, N.; Zhu, K.; Kjoniksen, A.L.; Knudsen, K.D.; Karlsson, G.; Nystroem, B. Effect of temperature and ph on the contraction and aggregation of microgels in aqueous suspensions. J. Phys. Chem. B 2009, 113, 11115-11123.

16. Zhou, S.; Chu, B. Synthesis and volume phase transition of poly(methacrylec acid-co-N-isopropylacrylamide) microgel particles in water. J. Phys. Chem. B 1998, 102, 1364-1371.

17. Hoare, T.; Pelton, R. Functional group distributions in carboxylic acid containing poly(N-isopropylacrylamide) microgels. Langmuir 2004, 20, 2123-2133.

18. Karg, M.; Pastoriza-Santos, I.; Rodriguez-Gonzalez, B.; von Klitzing, R.; Wellert, S.; Hellweg, T. Temperature, $\mathrm{pH}$, and ionic strength induced changes of the swelling behavior of PNIPAM-Poly(allylacetic acid) copolymer microgels. Langmuir 2008, 24, 6300-6306.

19. Hoare, T.; Pelton, R. Highly $\mathrm{pH}$ and temperature responsive microgels functionalized with vinylacetic acid. Macromolecules 2004, 37, 2544-2550.

20. Hoefl, S.; Zitzler, L.; Hellweg, T.; Herminghaus, S.; Mugele, F. Volume phase transition of "smart" microgels in bulk solution and adsorbed at an interface: A combined AFM, dynamic light, and small angle neutron scattering study. Polymer 2007, 48, 245-254.

21. Fernandez-Nieves, A.; Fernandez-Barbero, A.; Vincent, B.; de las Nieves, F.J. Charge controlled swelling of microgel particles. Macromolecules 2000, 33, 2114-2118.

22. Pelton, R.H.; Chibante, P. Preparation of aqueos latices with $\mathrm{N}$-isopropylacrylamide. Colloid Surf. 1986, 20, 247-256.

23. Provencher, S.W. Inverse problems in polymer characterization: Direct analysis of polydispersity with photon correlation spectroscopy. Makromol. Chem. 1979, 180, 201-209. 
24. Burmistrova, A.; Richter, M.; Uzum, C.; von Klitzing, R. Effect of cross-linker density of $\mathrm{P}$ (NIPAM-co-AAc) microgels at solid surfaces on the swelling/shrinking behaviour and the Youngs modulus. Colloid Polym. Sci. 2011, 289, 613-624.

25. Dimitriadis, E.; Horkay, F.; Maresca, J.; Kachar, B.; Chadwick, R. Determination of elastic moduli of thin layers of soft material using the atomic force microscope. Biophys. J. 2002, 82, 2798-2810.

26. Larsson, A.; Kuckling, D.; Schoenhoff, M. 1H NMR of thermoreversible polymers in solution and at interfaces: The influence of charged groups on the phase transition. Colloid Surf. A 2001, 190, 185-192.

27. Hashmi, S.M.; Dufresne, E.R. Mechanical properties of individual microgel particles through the deswelling transition. Soft Matter 2009, 5, 3682-3688.

28. Cheng, X. Surface chemical and mechanical properties of plasma-polymerized $\mathrm{N}$-isopropylacrylamide. Langmuir 2005, 21, 7833-7841.

29. Daly, E.; Saunders, B.R. Temperature-dependent electrophoretic mobility and hydrodynamic radius measurements of poly(N-isopropylacrylamide) microgel particles: Structural insights. Phys. Chem. Chem. Phys. 2000, 2, 3187-3193.

30. Burmistrova, A.; Steitz, R.; von Klitzing, R. Temperature response of PNIPAM derivatives at planar surfaces: Comparison between polyelectrolyte multilayers and adsorbed microgels. ChemPhysChem 2010, 11, 3571-3579.

(c) 2011 by the authors; licensee MDPI, Basel, Switzerland. This article is an open access article distributed under the terms and conditions of the Creative Commons Attribution license (http://creativecommons.org/licenses/by/3.0/.) 